\title{
Molecular Dynamics Study of Thermodynamic Scaling of the Glass-Transition Dynamics in Ionic Liquids over Wide Temperature and Pressure Ranges
}

\author{
J. Habasaki, ${ }^{* \dagger}$ R. Casalini, ${ }^{\ddagger}$ and K. L. Ngai ${ }^{\ddagger}$ \\ Tokyo Institute of Technology, Nagatsuta 4259, Yokohama 226-8502, Japan, and Naval Research Laboratory, \\ 4555 Overlook Avenue SW, Washington, DC 20375-5320
}

Received: November 24, 2009; Revised Manuscript Received: January 15, 2010

\begin{abstract}
Experimentally, superpositioning of dynamic properties such as viscosity, relaxation times, or diffusion coefficients under different conditions of temperature $T$, pressure $P$, and volume $V$ by the scaling variable $T V^{\gamma}$ (where $\gamma$ is a material constant) has been reported as a general feature of many kinds of glass-forming materials. In the present work, molecular dynamics (MD) simulations have been performed to study the scaling of dynamics near the glass-transition regime of ionic liquids. Scaling in the simulated 1-ethyl-3methylimidazolium nitrate $\left(\mathrm{EMIM}-\mathrm{NO}_{3}\right)$ system has been tested over wide ranges of temperatures and pressures. $T V^{\gamma}$ scaling of the dynamics is well described by master curves with $\gamma=4.0 \pm 0.2$ and $3.8 \pm 0.2$ for cation and anion, respectively. Structures and Coulombic terms of the corresponding states are found to be quite similar. The temperature and pressure dependence of the pair correlation function show similar trends and therefore can be superpositioned onto the master curve. Although the behaviors with $\gamma=4$ might be expected from the relation, $\gamma=n / 3$, for the dynamics with the soft-core-type potential $U=\varepsilon(\sigma / r)^{n}$, with $n$ $=12$, pair potentials used in the MD simulation have a more complex form, and not all the repulsive terms can play their roles in the heterogeneous structures determined by ion-ion interactions. Scaling is related to the common part of effective potentials related to the pair correlation functions, including the many-body effect in real space.
\end{abstract}

\section{Introduction}

Complex relaxations in supercooled liquids and their glass transitions are important long-standing problems of condensed matter physics and chemistry. ${ }^{1-4}$ Although supercooled liquids and glasses including ionic systems show complicated heterogeneous dynamics, ${ }^{5-7}$ an existence of a simple scaling behavior of the dynamic properties, $x=\mathscr{T}\left(T V^{\prime}\right)$ (where $T$ is temperature and $V$ is the specific volume), has been reported for many systems, ${ }^{8-11}$ where $x$ can be the relaxation time $\tau$ or viscosity $\eta$, while $\gamma$ is a material constant.

In the present work, we have compared the structures and energies of the various points on the master curve, which show similar diffusion coefficients. In the case of simpler systems with the soft-core potential, $U=\varepsilon(\sigma / r)^{n}$, or Lennard-Jones-type potentials, scaling is related to the local slope on the repulsive part of the potential near the potential minimum and has been explained to originate from the repulsive softness of the potential parameters used in the simulation. ${ }^{12,13}$

A class of materials called room-temperature ionic liquids, or simply ionic liquids (ILs), whose melting points are below or near room temperature, ${ }^{14-17}$ has attracted much attention in the research community because they possess many desirable properties for applications such as high thermal stability and negligible vapor pressure. Recently, successful $T V^{\gamma}$ scaling of viscosity in ionic liquids has been also reported, ${ }^{9,11}$ where $\gamma$ is found to be at around 2-4. The structures of systems are determined by the combinations of cation-cation, cation-anion, and anion-anion interactions, and the scaling parameter $\gamma$ is

* To whom correspondence should be addressed. E-mail: habasaki.j.aa@m.titech.ac.jp.

$\uparrow$ Tokyo Institute of Technology.

* Naval Research Laboratory. different from the $r^{-1}$ dependence of the Coulombic term. The purpose of the present work is to examine the relation between structures and potential parameters and other factors that affect $\gamma$ by means of MD simulations in a complex system such as an ionic liquid. Changes in the structure with temperature and pressure conditions also are examined in details.

\section{Molecular Dynamics Simulations}

We study the dynamics of the IL 1-ethyl-3-methyl-imidazolium nitrate $\left(\mathrm{EMIM}-\mathrm{NO}_{3}\right.$ ) by using the potential functions of the following form

$$
\begin{gathered}
U(r)=\sum_{\text {bonds }} K_{\mathrm{r}}\left(r-r_{\mathrm{eq}}\right)^{2}+\sum_{\text {angles }} K_{\theta}\left(\theta-\theta_{\mathrm{eq}}\right)^{2}+ \\
\sum_{\text {dihedrals }} \frac{V_{\mathrm{n}}}{2}\left(1+\cos \left[n \phi-\gamma^{\prime}\right]\right)+\sum_{i<j}^{\text {atoms }}\left(\frac{A_{i j}}{r_{i j}^{12}}-\frac{B_{i j}}{r_{i j}^{6}}\right)+ \\
\sum_{i<j}^{\text {atoms }} \frac{q_{i} q_{j}}{\varepsilon r_{i j}}
\end{gathered}
$$

similar to those used in previous works of ILs. ${ }^{18-24}$ It is a sum of bond, angle, and dihedral deformation energies, the pairwise standard $(6,12)$ Lennard-Jones potential representing the repulsive term and van der Waals interactions, and Coulombic interactions between atoms with charges $q_{i}$. The parameters therein were taken from the general Amber force field. ${ }^{25}$ The program AMBER (ver. 9) ${ }^{26}$ was used. The equilibrated density was $1.206 \mathrm{~g} \mathrm{~cm}^{-3}$ at $400 \mathrm{~K}$ under atmospheric pressure. The value is consistent with the experimental value of $1.28 \mathrm{~g} \mathrm{~cm}^{-3}$ at $293 \mathrm{~K}^{27}$ from consideration of the temperature dependence of the density. ${ }^{23}$ In the model system, the charge for each cation 
and anion is assumed to be +1 and -1 , respectively, while partial charges are used on each atom. As shown later, the Coulombic terms have importance in describing the dynamics such as diffusion coefficients of the system and modification of the formal charges on each ion and/or introducing a polarizability term, which may be necessary for the quantitative description of the system. This is also consistent with the discussion previously given in ref 23 . Further examination for the parameters in the repulsive terms is also necessary. Despite these problems, the present system can be regarded as the useful model for consideration of general properties of ILs such as scaling and glass transition. That is, a model of ionic liquids in MD simulations is not necessarily fully representative of the real system but is a valid physical system worth examining.

MD simulations were performed using this force field on smaller systems having $64 \mathrm{EMIM}^{+}$and $64 \mathrm{NO}_{3}{ }^{-}$with a total of 1472 atoms and the larger system having $256 \mathrm{EMIM}^{+}$and $256 \mathrm{NO}_{3}{ }^{-}$with a total of 5888 atoms. Results of both systems will be used for discussion since the system size dependence of the diffusivity of the system is found to be small, except for some details. Periodic boundary conditions were imposed, and Coulomb interactions were calculated using the particle mesh Ewald method. The system was equilibrated at $3000 \mathrm{~K}$, and from there, the temperature was gradually decreased.

The time step was either 1 or $2 \mathrm{fs}$ in the simulations of the $N V E$ or NPT ensembles (using the weak coupling by Berendsen ${ }^{28,29}$ ) that were carried out up to 2.5 or $10 \mathrm{~ns}$ after enough equilibration time of several nanoseconds at each temperature. The diffusion coefficients obtained in the NPT runs under atmospheric conditions are comparable to those obtained for the $N V E$ ensemble. Temperature and pressure conditions used for the check of the scaling behaviors will be shown later. Although not necessarily required to show the scaling behaviors, additional data points are used in some figures due to the following reasons. (1) In some analyses, a larger number of data points is required to reduce the error of the parameter fitting or summations. (2) Possible covered regions are not the same among thermal, structural, and transport properties. Especially, at low temperature or under high pressure regions, long-time MD runs are required to attain a diffusive regime, while a shorter time scale run was enough to obtain the thermal or structural properties.

\section{Results and Discussion}

Experimentally, the melting point of the present ionic liquid is known to be $313 \mathrm{~K}$, and crystallization occurs at about 290 $\mathrm{K} .{ }^{27}$ Although the system is known to show crystallization, by rapidly quenching in the simulation $\left(\sim 1^{\circ} / \mathrm{ps}\right)$, a change of the slope is found in the plot of density versus temperature. The glass-transition temperature of the simulated system determined from the change is $260 \mathrm{~K}$.

III.A. Scaling Based on the Diffusion Coefficient. Diffusion coefficients were obtained from the long run at each condition using the Einstein equation after the subdiffusion regime. Complicated behavior for the mean-square displacment (MSD) in several time regions is well-known in ionic systems such as ionically conducting glasses. ${ }^{30} \mathrm{~A}$ similar situation has been found in several ionic liquids. ${ }^{18,19,23}$ Examples of the MSD for EMIM ${ }^{+}$ ions in several conditions are shown in Figure 1a. Solid (blue) curves are for the data in $\sim 0.1 \mathrm{MPa}$ at several temperatures. A dash-dotted (pink) curve is for the data at $510 \mathrm{~K}(500 \mathrm{MPa})$, and a dashed curve (green) is for the data at $800 \mathrm{~K}(1 \mathrm{GPa})$.

Some caution is required for the analysis at lower temperatures. At the lowest temperature of $300 \mathrm{~K}$, in the short-time region, the MSD increases weakly with time because the
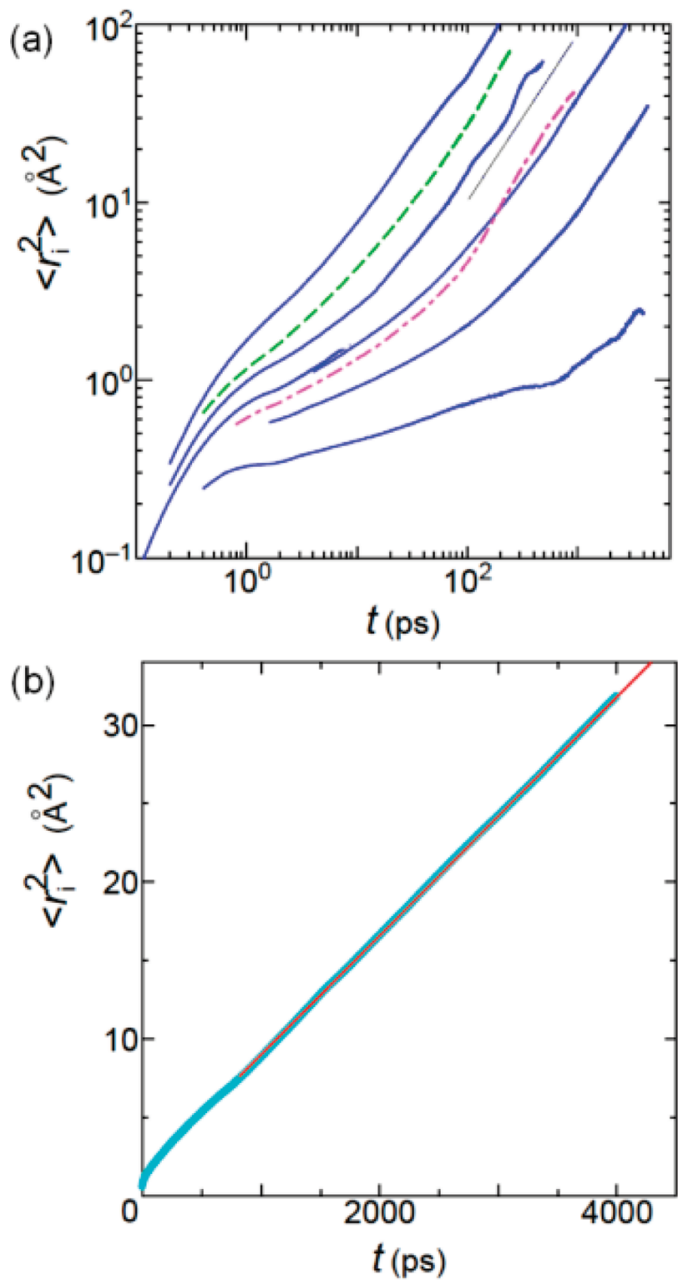

Figure 1. Mean-squared displacements of the $\mathrm{EMIM}^{+}$ion for the center of masses motion in EMIM- $\mathrm{NO}_{3}$ under several temperature and pressure conditions. (a) Solid (blue) curves are for the data in $\sim 0.1$ $\mathrm{MPa}$ at $600,500,400,370$, and $300 \mathrm{~K}$. A dash-dotted (pink) curve is for the data at $510 \mathrm{~K}(500 \mathrm{MPa})$, and a dashed curve is for the data at $800 \mathrm{~K}(1 \mathrm{GPa})$. (b) Plot of the data at $370 \mathrm{~K}(\sim 0.1 \mathrm{MPa})$ (circles, pale blue) using the linear scales. A red line is for the fitted one, which is proportional to time.

$\mathrm{EMIM}^{+}$ions are caged, and it corresponds to the nearly constant loss $(\mathrm{NCL})^{31-34}$ regime observed in the dielectric loss by a conductivity relaxation experiment. This caged regime extends to longer times, and the diffusive regime is not attained up to the longest time of the simulation. Therefore, such data at low temperatures are omitted from further consideration of scaling. The black line in Figure 1a is for the slope 1 on the double logarithmic scales, and the deviation from it at a shorter time scale is usually regarded as the deviation from the normal diffusion. In Figure 1a, the slope becomes 1 for high-temperature data at long times. However, a deviation from the slope 1 is found at long time (after $\sim 1 \mathrm{~ns}$ ) even after the subdiffusive regime at $370 \mathrm{~K}$. A corresponding plot of the MSD on the linear scale (Figure $1 \mathrm{~b}$ ) is proportional to time after $\sim 1 \mathrm{~ns}$. In this case, the deviation on the double logarithmic scale is not due to the different time regions but is caused by overlap of the short-time behavior. Therefore, the data at $370 \mathrm{~K}$ and similar data can be used to calculate the diffusion coefficients by the Einstein equation. In this case, 10000000 steps run with $\delta t=$ $1 \mathrm{fs}$ and many initial times (>10000) over wide time windows were used for averaging.

In Figure 2a, diffusion coefficients for $\mathrm{EMIM}^{+}$are plotted against inverse temperature. The temperature dependence of 


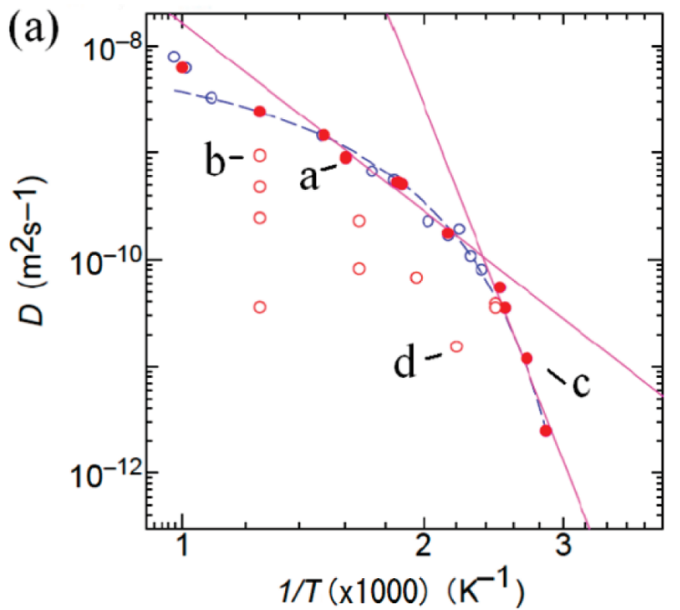

(b)
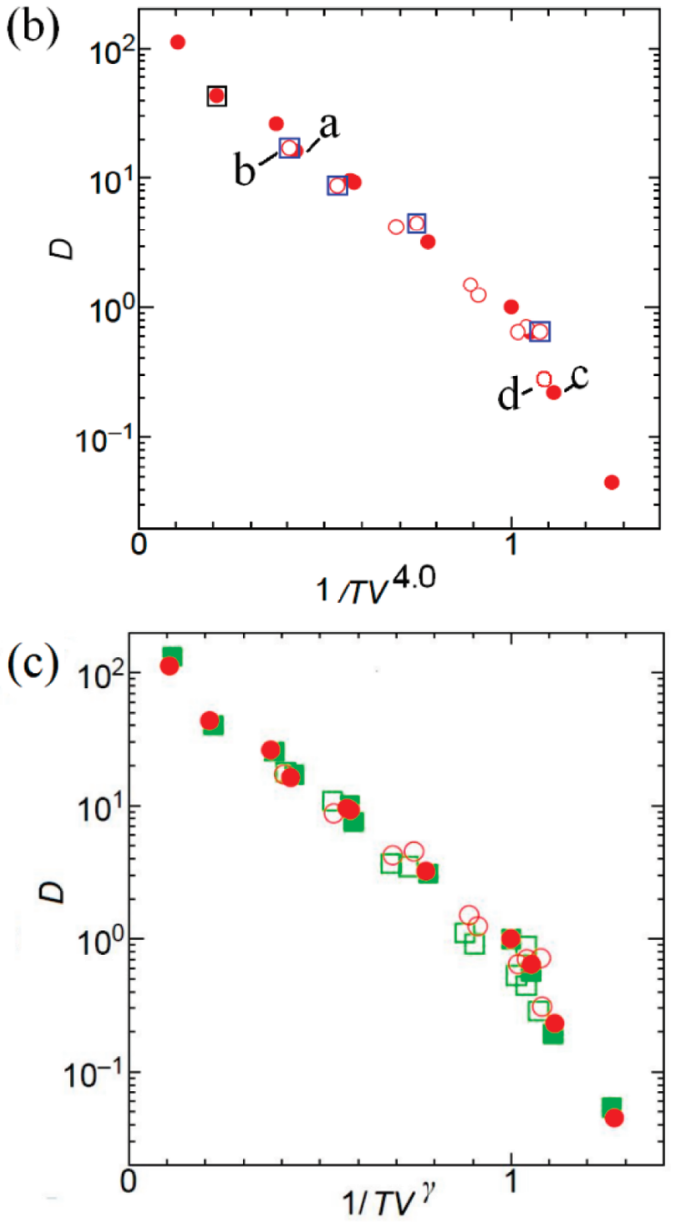

Figure 2. (a) The temperature dependence of the diffusion coefficient of $\mathrm{EMIM}^{+}$for the center of masses motion in $\mathrm{EMIM}-\mathrm{NO}_{3}$ is shown by solid (red) circles. A dashed curve (blue) is fitted to the Vogel-Fulcher-Tammann (VFT) type, while solid lines (pink) are for power law type functions. Additional data points (open circles, blue) are also used for fitting. Open (red) circles are data under pressure. (b) Diffusion coefficients in (a) are plotted against $1 / T V^{4}$. Isothermal data at $800 \mathrm{~K}$ are shown by open red circles within open blue squares. Data are normalized for $400 \mathrm{~K}$ under $\sim 0.1 \mathrm{MPa}$, which is near the inflection point of the diffusivity. Two sets of data, (a) $(600 \mathrm{~K}, \sim 0.1 \mathrm{MPa})$ and (b) $(800 \mathrm{~K}, 500 \mathrm{MPa})$ and (c) $(370 \mathrm{~K}$, $\sim 0.1 \mathrm{MPa}$ ) and (d) $(450 \mathrm{~K}, 500 \mathrm{MPa})$ shown in these Figures are compared in detail. (c) Scaled plots against $1 / T V^{\prime}$ of the diffusivity for the anion. Data are normalized for $400 \mathrm{~K}$ under $\sim 0.1 \mathrm{MPa}$. Filled green squares are for the temperature dependence, and open green squares are under pressures. The data for the cations are again shown for the sake of comparison, where the marks are the same as those in (a) and (b). The scaling behavior for the anion is similar to that of cation; however, some differences are observed, as mentioned in the text. the diffusion coefficients at ambient pressure $(\sim 0.1 \mathrm{MPa})$ is shown by the solid (red) filled circles. The best fit to the Vogel-Fulcher-Tamman (VFT) equation ${ }^{35}$ is shown in the plot (dashed curve (blue) in Figure 2a). We used additional data points shown by blue circles in Figure 2a for fitting.

The best-fit parameters for $D=D_{0} \exp \left[E /\left(T-T_{0}\right)\right]$ obtained are $D_{0}=1.22 \times 10^{-8}\left(\mathrm{~m}^{2} \mathrm{~s}^{-1}\right), E=-907.69(\mathrm{~K})$, and $T_{0}=$ $243.24(\mathrm{~K})$. Power law fits with inflection are also shown by solid (pink) lines.

The best-fit parameters obtained in the form of $\ln (D)=A_{0}$ $+A_{1}(1000 / T)$ are $A_{0}=-17.94$ and $A_{1}=-5.78$ for the hightemperature regime and $A_{0}=-6.44$ and $A_{1}=-19.05$ for the low-temperature regime, where $D$ is in $\mathrm{m}^{2} \mathrm{~s}^{-1}$ and $T$ is in $\mathrm{K}$. The data under high pressure are shown by open (red) circles.

The validity of the scaling behavior $D=\mathscr{T}\left(T V^{\prime}\right)$ of the data shown in Figure 2a is demonstrated in Figure 2b, where the data are plotted against $1 / T V^{\prime}$, with $\gamma=4.0$. In this plot, data points at $800 \mathrm{~K}$ at $0.1,500,1000,2000$, and $4000 \mathrm{MPa}$ are represented by open red circles within open blue squares. All data points are found to fall on a master curve; therefore, diffusion coefficients are well scaled with $\gamma=4.0 \pm 0.3$. The data for the anions are plotted in Figure 2c. The data for the cations are again shown for the sake of comparison. A similar $T V^{\gamma}$ relation for the diffusivity for the anion with a slightly smaller $\gamma$ value $(=3.8)$ is obtained. Therefore, scaling holds for both of them, except for a small deviation discussed below. This can be expected from the similar behavior ${ }^{18,23}$ of diffusion coefficients of $\mathrm{EMIM}^{+}$and $\mathrm{NO}_{3}{ }^{-}$ions. In the temperature dependence under 0.1 MPa, the cation (filled red circles) and anion (filled green squares) behave in a similar manner, while in the pressure dependence, cations (open red circles) are slightly faster than anions (open green squares) near an inflection in the plots. Small deviation from the scaling for anions seems to be related to it. That is, the data for anions under pressure (open green squares) show a smaller curvature (fragility) compared with the other data points in Figure 2c.

As shown in previous works, ${ }^{23,24}$ the slope of the temperature dependence of the diffusion coefficient changes at around 400 $\mathrm{K}$ in the double logarithmic plot, and a similar situation is also observed in a semilogarithmic plot. The master curve also shows some curvature or inflection, and similar features are observed for other systems in both experiments and simulations. ${ }^{9,10,13}$ To examine the change in the dynamics, we have studied the trajectories of the ions. They show complexity reflecting the geometrical correlation among successive motions. ${ }^{24,36}$ Fractal dimension of the random walk is a measure of this complexity, and the rapid increase of the dimension upon decreasing temperature was found. Inflection in the diffusion coefficient has been explained as the change from the long-range (accelerated) to short-length-scale motion. ${ }^{24}$ The long-range motion is related to the cooperative motion of ions found in the heterogeneous dynamics. ${ }^{7}$ This explanation is valid for the master curve, and it means that the scaling describes both short- and long-ranged motion. Two sets of data points characterized by nearly the same value of $D$ at different combinations of $T$ and $P$ (a-b pair and $\mathrm{c}-\mathrm{d}$ pair) located nearby in the master curve in Figure $2 b$ are compared in the following section. These pairs are located in different regimes of the master curve.

The exponent obtained for $\mathrm{EMIM}^{+}-\mathrm{NO}_{3}{ }^{-}$is similar to the value for the soft-core potential ${ }^{37,38}$ with $n=12$; that is, $\gamma=$ $n / 3$. Of course, there can be relations between the repulsive parameters used and the scaling results; however, the repulsive term with $r^{-12}$ dependence in eq 1 is not for ion-ion interaction of the molecular ions but is for each atom-atom interaction. 


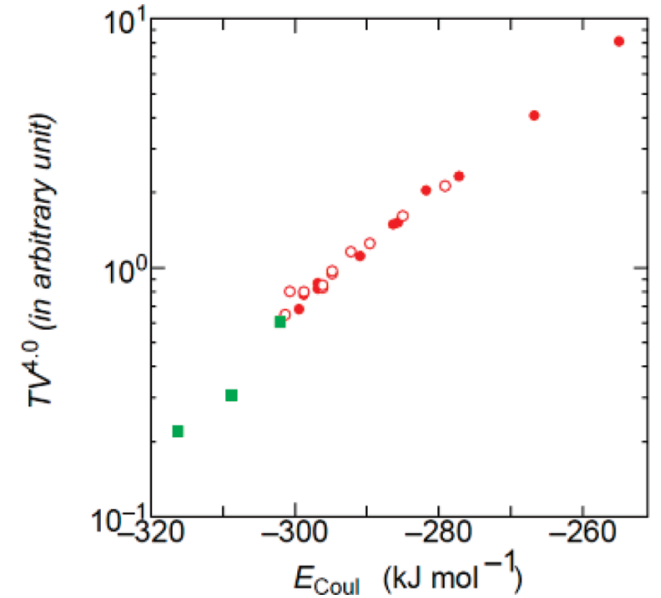

Figure 3. Plot of $T V^{4}$ against the Coulombic energy of the system. The master curve is found to be stable in the plot. Filled circles (red): Temperature dependence under $\sim 0.1 \mathrm{MPa}$. Open circles (red): under high pressures at several temperatures. Filled squares (green): lowtemperature data under $\sim 0.1 \mathrm{MPa}$. Corresponding points are not shown in Figure 2 because diffusive regimes were not obtained during the observation time.

All atoms cannot respond to the scaling in the complicated system with heterogeneity, where ion-ion interactions play important roles. In the present work, we have examined the effective potential among ions by considering the structure due to the ion-ion interactions, as well as the importance of the Coulombic term to be shown later. The underlying effective potential for ion-ion interaction that is concerned with the scaling behaviors is expected to be similar to the inverse power law type of potential rather than the $r^{-1}$ dependence of the electrostatic term. From the different experimental values of $\gamma$ for other ILs, ${ }^{9,11} n$ values different from 12 seems to be possible. Despite the similarity to the soft-core model, it is necessary to examine carefully the origin of the scaling in these more complex systems.

Thus, we could reproduce the scaling behaviors as found in the experiments. The exponent is comparable or larger than experimentally found values for related systems. We have examined what part of the potential parameters is important for the scaling.

III.B. Comparison between Corresponding States on a Master Curve. In this section, we will show what part of the energy term is related to the scaling. Energy terms at $400 \mathrm{~K}$ obtained from MD are comparable with those reported by Popolo and Voth ${ }^{17}$ obtained by using slightly different potential parameters. The Coulombic energy $\left(E_{\text {Coul }}\right)$ and total potential energy $\left(E_{\text {ptot }}\right)$, which is a sum of terms in eq 1 , for $\mathrm{a}-\mathrm{b}$ and $\mathrm{c}-\mathrm{d}$ pairs are compared. We find that the Coulombic energy for a $(600 \mathrm{~K}, \sim 0.1 \mathrm{MPa}), E_{\text {Coul }}=-281.15 \mathrm{~kJ} \mathrm{~mol}^{-1}$, is comparable to that for b $(800 \mathrm{~K}, 500 \mathrm{MPa}), E_{\mathrm{Coul}}=-278.92$ $\mathrm{kJ} \mathrm{mol}^{-1}$, while the potential energy of a, $E_{\mathrm{ptot}}=-139.68 \mathrm{~kJ}$ $\mathrm{mol}^{-1}$, is considerably smaller than that for $\mathrm{b}, E_{\mathrm{ptot}}=-93.31$ $\mathrm{kJ} \mathrm{mol}^{-1}$.

For the other pair, we found that the Coulombic energies, $E_{\text {Coul }}$, of c $\left(-299.0 \mathrm{~kJ} \mathrm{~mol}^{-1}\right.$ at $\left.370 \mathrm{~K}, 0.1 \mathrm{MPa}\right)$ and d (-298.6 $\mathrm{kJ} \mathrm{mol}^{-1}$ at $\left.450 \mathrm{~K}, 500 \mathrm{MPa}\right)$ are comparable. $E_{\mathrm{ptot}}$ for c $(-228.6$ $\left.\mathrm{kJ} \mathrm{mol}^{-1}\right)$ is again different from that for $\mathrm{d}\left(-207.6 \mathrm{~kJ} \mathrm{~mol}^{-1}\right)$. This result suggests that the Coulombic term plays a major role in the scaling behaviors of ionic liquids.

As shown in Figure 3, when $T V^{\gamma}(\gamma=4.0)$ is plotted against the electrostatic energy of the system, the master curve remains almost stable. That is, the systems in corresponding states on the master curve have comparable electrostatic energies. For the data corresponding to those in Figure 2, there is no significant change in the slope. Low-temperature data under $\sim 0.1 \mathrm{MPa}(330,200$, and $150 \mathrm{~K})$ are also plotted in Figure 3 (filled squares, green), where diffusive regimes were not obtained during the observation time. The slope for the relation between the electrostatic energy and $T V^{\prime}$ changes near the glasstransition point $(\sim 260 \mathrm{~K})$.

To perform the NVT ensemble simulation, one may prefer the method by Nosé, ${ }^{39}$ which ensures the formation of a canonical ensemble. Related methods such as Nosé-Andersen ${ }^{40}$ may be also used for NPT ensemble simulations. However, we used the weak coupling method to simulate the situation near the glass transition in the present work. This is because the extended methods with additional degrees of freedom might affect the non-Gaussian dynamics ${ }^{17,22}$ found in ionic liquids. This problem is common in many systems such as ionics in glasses and supercooled liquids near the glass-transition regimes. Further study may be necessary to obtain the difference among different ensembles, including the algorithm employed.

In Figure 4, pair correlation functions, $g(r)$, of $\mathrm{EMIM}^{+}-$ $\mathrm{NO}_{3}{ }^{-}, \mathrm{NO}_{3}{ }^{-}-\mathrm{NO}_{3}{ }^{-}$, and $\mathrm{EMIM}^{+}-\mathrm{EMIM}^{+}$pairs are compared for the $\mathrm{a}-\mathrm{b}$ pair and the $\mathrm{c}-\mathrm{d}$ pair in Figure 2. Correspondence is good for both cases. That is, the corresponding states have comparable pair correlation functions. Correspondence seems to be excellent especially for a cation-anion pair. Some differences found in other pairs suggest that the correspondence in the master curve is mainly governed by the cation-anion interaction with the shortest distance, and the small differences in cation-cation and anion-anion pairs are not important for the scaling, at least for the present system. The correspondence holds for a limited $r$ region and does not hold for a larger $r$ region because of the different volumes of the systems in these pairs. The height of the first peak in the cation-anion pair in Figure 2a is smaller than that in Figure 2b. This is consistent with the large difference of the potential and Coulombic energies. One can find some experimentally obtained $g(r)$ 's under high-pressure conditions in heterogeneous liquids under high pressure $(\sim \mathrm{GPa})^{41}$ or in ionic liquids under moderate pressures $^{42}(\sim \mathrm{MPa})$.

Thus, we found several characteristics of scaling behaviors summarized as follows. Similarity in the electrostatic term is observed when similar behavior on the master curve is found, while a common exponent controlling the system has an inverse power law form. Correspondence in the electrostatic term is related to the similarity of the pair correlation functions for ion-ion pairs. Scaling is related to both short- and long-lengthscale motions.

III.C. Temperature and Pressure Dependences of Structures. In Figure 5a, the pressure dependence of $g(r)$ at $800 \mathrm{~K}$ is shown for cation-anion, anion-anion, and cation-cation pairs, calculated using a center of mass position of each ion. Additionally, two high-pressure conditions (5000 and $6000 \mathrm{MPa}$ ) are used in Figure 5a. The temperature dependence of the functions is shown in Figure 5b. In both cases, the distance $r$ is scaled by the mean distance of the same kind of ions, $L$, which is proportional to the length of the simulation box. As already shown for the two cases, the systems in the corresponding states have similar structures. One can expect the system with different conditions to behave in a similar manner if $T V^{\gamma}$ is the same. The comparison by scaled $r$ is useful to distinguish the changes in peaks in the first coordination shell. The shape of the first peaks is found to change, especially in the high-pressure and low-temperature region. The position of the first peak shifts 

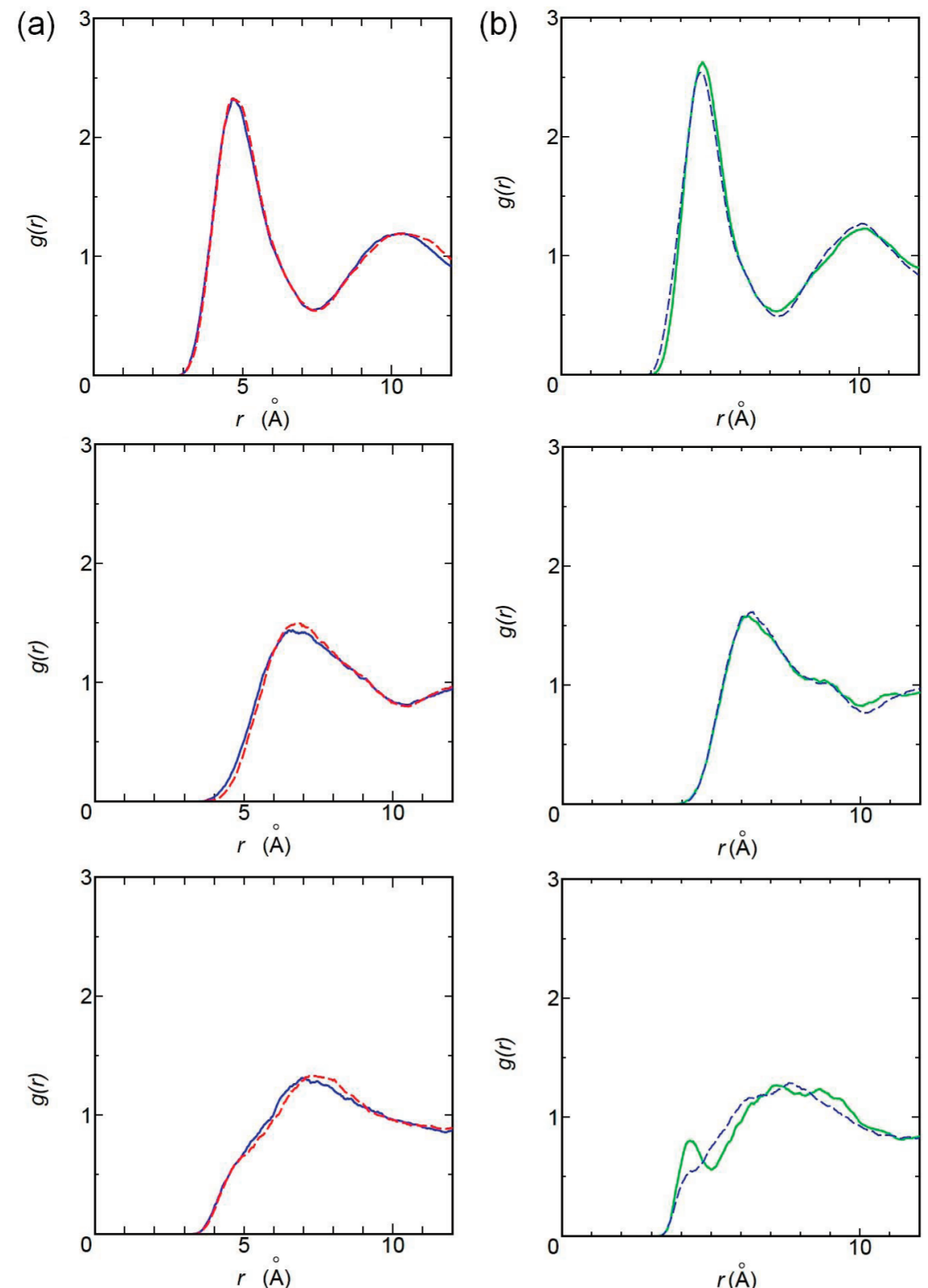

Figure 4. Comparison of the structure of the systems in states having similar $D$ on the master curve. Pair correlation functions of $\mathrm{EMIM}^{+}-\mathrm{NO}_{3}{ }^{-}$, $\mathrm{NO}_{3}{ }^{-}-\mathrm{NO}_{3}{ }^{-}$, and $\mathrm{EMIM}^{+}-\mathrm{EMIM}^{+}$from top to bottom for Figure $2 \mathrm{a}$ (red dashed curve) and Figure $2 \mathrm{~b}$ (blue, continuous curve). The function for the $\mathrm{EMIM}^{+}-\mathrm{NO}_{3}^{-}, \mathrm{NO}_{3}^{-}-\mathrm{NO}_{3}^{-}$, and $\mathrm{EMIM}^{+}-\mathrm{EMIM}^{+}$pairs for Figure $2 \mathrm{c}$ (green, thick curve) and Figure $2 \mathrm{~d}$ (blue, dashed curve).

toward larger $r$ with increasing pressure at a fixed temperature. A similar trend is found for the temperature dependence with decreasing temperature. Scaling by $L$ might be exact if the structure is self-similar; however, this is not the case. The compressibility of the structure with short distance seems to be different from that for the long-ranged one. The peak height tends to decreases once and then increases again in both a and $\mathrm{b}$ in Figure 5. Changes compensating for those in the first shell exist in the second and sequential peaks.

For a cation-cation pair, the shoulder of short distance $(\sim 0.6$ $(4 \AA)$ ) is found in both Figure $5 \mathrm{a}$ and b. This component was previously assigned for a pair with parallel imidazolium rings. ${ }^{24}$ With increasing pressure at $800 \mathrm{~K}$, the shoulder becomes clearer and shows a maximum at around $5000 \mathrm{MPa}$. A similar maximum is found in the temperature dependence. The shoulder seems to be largest at around $370 \mathrm{~K}$ under $\sim 0.1 \mathrm{MPa}$. In parallel, changes in the heights of the first peaks are observed. Distortion in the shape of the first peak is found, especially at low temperatures and under high pressure. It is interesting to note that both the smallest height of the first peak in the cation-anion pair and the largest contribution of the shoulder in cation-cation pairs are observed near the inflection points of the master curve. Changes in the longer-ranged structure are found near the inflection point, and details will be reported elsewhere.

Similarity of the $g(r)$ of systems under different conditions is remarkable for the first peak of the cation-anion interaction in the real units rather than in the scaled units. Gradual changes in the slopes common for increasing temperature and pressure exist; however, their effects on the scaling exponents are small if any. Thus, the correspondence holds well, including the appearance of the sub peaks or the distortion of peaks, except for some minor details.

As an aside, it is interesting to note that the dynamics in the nonionic glass-forming liquid, ortho-terphenyl, follow the $T V^{\gamma}$ scaling, and the similarity in the structure factors in neutron scattering experiments under isochronal conditions has been observed under high pressures. ${ }^{8}$ 

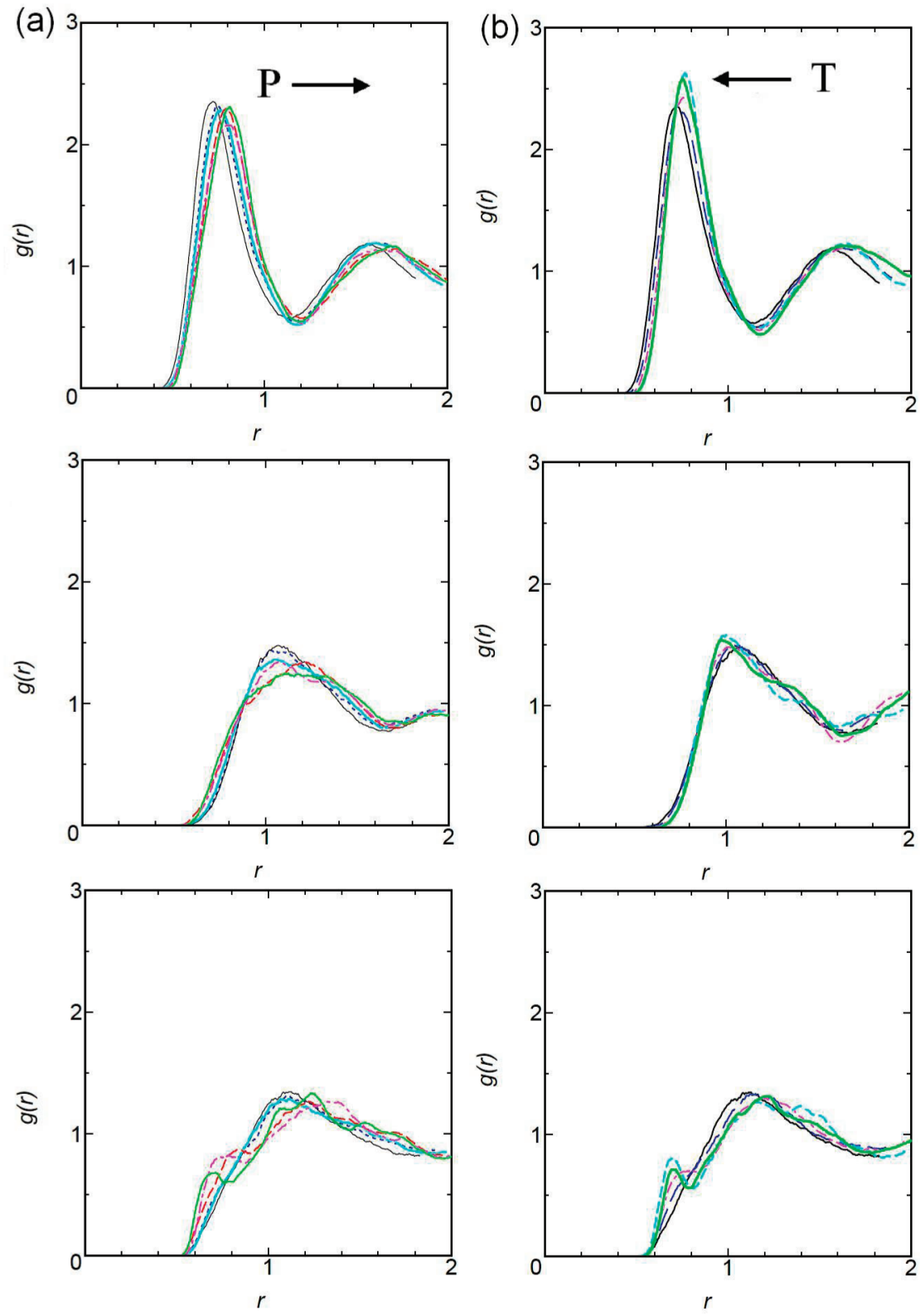

Figure 5. (a) Pressure dependence of the pair correlation function, $g(r)$, at $800 \mathrm{~K}$.Thin (black) curve: $0.1 \mathrm{MPa}$. Dotted (blue) curve: $500 \mathrm{MPa}$. Thick (pale blue) curve: $1000 \mathrm{MPa}$. Dash-dotted (pink) curve: $4000 \mathrm{MPa}$. Dashed (red) curve: $5000 \mathrm{MPa}$. Dash-dotted with larger intervals (green) curve: $6000 \mathrm{MPa}$. The distance is scaled by the mean distance of like ions. (b) Temperature dependence of the pair correlation functions, $g(r)$, at $\sim 0.1 \mathrm{MPa}$. Thin (black) curve: $800 \mathrm{~K}$. Dotted (blue) curve: $600 \mathrm{~K}$. Dash-dotted (pink) curve: $400 \mathrm{~K}$. Dashed (pale blue) curve: $370 \mathrm{~K}$. Thick (green) curve: $250 \mathrm{~K}$. Similar changes are found in (a) and (b). The minimum of the peak height in the first peak of $g(r)$ for the cation-anion pair and the maximum intensity of the shoulder of short distance for the cation-cation pair are found at around the inflection point of the dynamics.Arrows in the figure mean the directions of increasing pressure and that of increasing temperature.

There are small differences in the shape of $g(r)$ for anion-anion pairs in Figure $5 \mathrm{a}$ and $\mathrm{b}$. The first peak tends to be separated into broader peaks under high pressure, while this trend is not clear in the temperature dependence. The change in the curvature of the $g(r)_{\text {anion-anion }}$ near the first peak is responsible for deviation from the scaling for anions because motion among anion sites is affected by it.

In the present work, a given pressure was reached within several hundred steps using a time step of $1 \mathrm{fs}$. From the similarity of the temperature and pressure dependence, it is suggested that the rate of compression is one of the important factors to control the glass transition. It will be interesting to examine if the crystallization will occur or not with a slower compression rate.

III.D. Potential of Mean Force. Comparison of the energies for the corresponding points in the master curve has revealed that the correspondence is mainly due to Coulombic energies and not for the total energy. Thus, the ion-ion level structures are more important rather than the inner structure of the molecular ions for the scaling behaviors. Naturally, the correspondence of the dynamics is based on the similarity in the ion-ion structures because the diffusion coefficient examined is for ions. Similarity found in the pair correlation functions means similarity in the potential of the mean force (PMF) $W_{i j}(r)$, introduced by Kirkwood, ${ }^{43}$ which is connected to the pair correlation function $g_{i j}(r)$ for ion-ion pair in the following form

$$
W_{i j}(r)=-k_{\mathrm{B}} T \ln g_{i j}(r)
$$

We have examined it by further analyses. The PMF is playing an important role in statistical mechanical theories of liquids, ${ }^{44-46}$ such as the reference interaction site model (RISM). ${ }^{47}$ 
The potential is an effective potential among ions. Actually, Soper ${ }^{48}$ has successfully derived the effective potential parameters of fluid from the site-site pair correlation function, and the methods are now widely used (see also section III.F for approaches using effective potentials). Caution should be taken for the many-body character of the potential if one uses it for simulations.

Although the MD simulation under pressure in the present work is performed in the NPT ensemble, the scaling is expected to hold for different ensembles such as $N V T$ or $N V E$ ensembles, at least in the liquid state. Here, we consider the Helmholtztype free energy, $F$, in a canonical $(N V T)$ ensemble as a first approximation.

Generally, for an $N$-particle system, the force acting for an $i$ th number of particles is represented as follows ${ }^{44-46}$

$$
\begin{aligned}
-\frac{\partial W}{\partial \mathbf{r}_{\mathrm{i}}}=\frac{k_{\mathrm{B}} T}{g(r)} \cdot \frac{\partial g(r)}{\partial \mathbf{r}_{\mathrm{i}}}= & \frac{\int \ldots \int\left(-\frac{\partial U_{N}}{\partial \mathbf{r}_{\mathrm{i}}}\right) \exp \left\{-\beta U_{N}\right\} \mathrm{d} \mathbf{r}_{n+1}, \ldots, \mathrm{d} \mathbf{r}_{N}}{\int \ldots \int \exp \left\{-\beta U_{N}\right\} \mathrm{d} \mathbf{r}_{n+1}, \ldots, \mathrm{d} \mathbf{r}_{N}}
\end{aligned}
$$

where $U_{N}$ is for the potential energy of $N$ particles and $\beta$ is an inverse of $k_{\mathrm{B}} T$. In the case of the pair potential, $n=2$.

If we use a configurational part of a partition function, $Q_{N}$, for $N$ particles

$$
Q_{N}=\int_{v} \ldots \int_{v} \exp \left\{-\beta U_{N}\left(\mathbf{r}_{1}, \mathbf{r}_{2}, \ldots, \mathbf{r}_{N}\right)\right\} \mathrm{d} \mathbf{r}_{1}, \mathrm{~d} \mathbf{r}_{2}, \ldots, \mathrm{d} \mathbf{r}_{N}
$$

the Helmholtz type free energy, $F$, is

$$
F=E-T S=-k_{\mathrm{B}} T \ln Q_{N}(V, T)
$$

where $E=U+K$. Here $K, E$, and $S$ represent the kinetic energy, internal energy, and entropy, respectively. As shown in eq 4, a concept of the PMF contains many-body effects a priori and is related to the free-energy term in eq 5 . In the low density limit, the PMF becomes comparable to a bare potential.

So far, many problems to calculate the free energy have been pointed out in the literature. If the system has large energy barriers, preventing an accurate sampling, special methods such as the umbrella sampling technique might be required. ${ }^{49}$ In our simulations, the function $g(r)$ is reproducible and accurate enough for the systems under the investigated conditions, and therefore, we calculate the PMF from the pair correlation function directly using eq 2.

The potentials, $W_{i j}(r)$, calculated for cation-cation, anionanion, and cation-anion pairs from the corresponding $\mathrm{g}_{i j}(r)$ for the center of masses of ions at $400 \mathrm{~K}$ are shown in Figure 6. The potential can be regarded as an effective potential among ions, as already mentioned.

The effective potential thus obtained has the power law dependence. The fit in the double logarithmic plot is shown in the inset. Since the linear regions are clearly found for every pair, it is not difficult to determine the power law exponents from the "equations of the lines" in the plot, even if the power law region is limited. It is natural to assume the power law type function because we are looking for the exponent to explain the scaling. The power law exponents of the cation-cation,

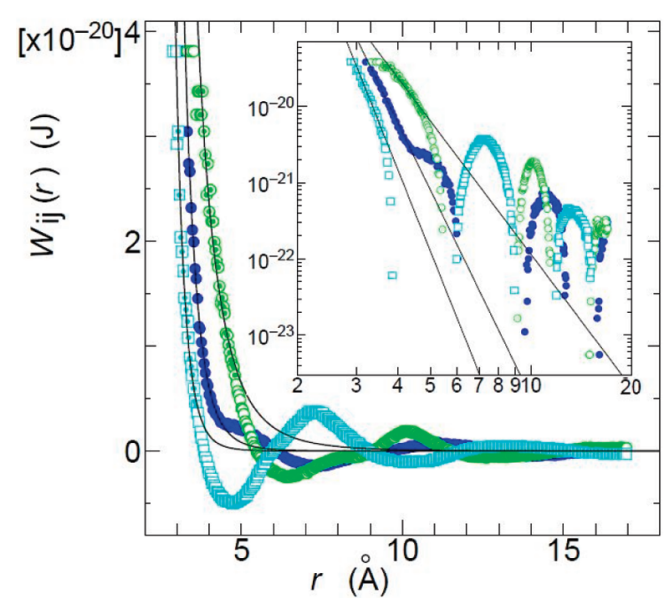

Figure 6. Potential of mean force between $\mathrm{EMIM}^{+}-\mathrm{NO}_{3}{ }^{-}$(pale blue), $\mathrm{NO}_{3}{ }^{-}-\mathrm{NO}_{3}{ }^{-}$(green), and $\mathrm{EMIM}^{+}-\mathrm{EMIM}^{+}$(blue) pairs calculated for the center of mass of each ion. Black curves are fitted curves of the power law function. A similar plot on a double logarithmic scale is shown in an inset. Existence of the power law regions is found clearly.

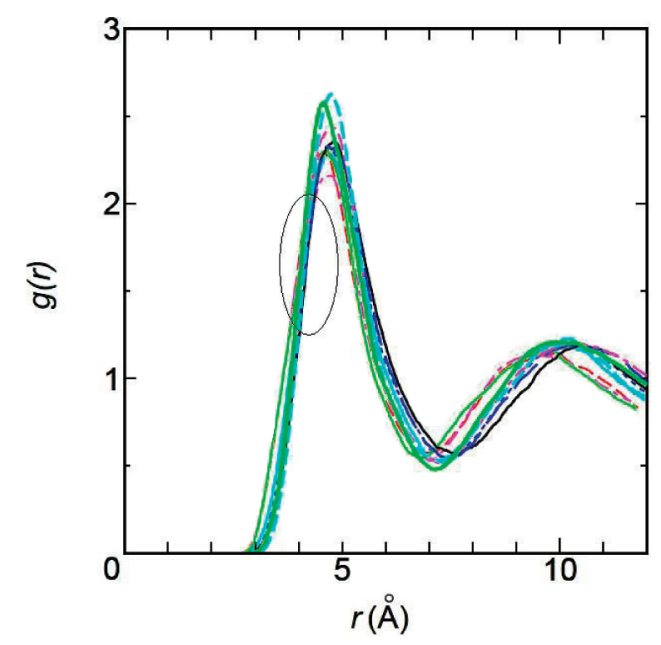

Figure 7. Pair correlation functions of different conditions as a function of $r(\AA)$. All data shown in Figure 5a and b for cation-anion pairs are included. Overlaps of slopes are found and marked by an oval.

anion-anion, and cation-anion are found to be $-9.0,-5.8$, and -11.0 , respectively. As a first approximation, it is probable that the $g(r)$ of the cation-anion pair having the shortest distance is important for the scaling including the compression of the system, although a small difference in $\gamma$ values between the cation and anion suggests the contribution of other parts of the structures to the scaling behaviors. From the slope for the cation-anion pair, $\gamma$ can be estimated by $(11.0 / 3) \sim 3.7$, which is near the value of the exponent equal to $4.0 \pm 0.3$ obtained by the scaling within a statistic error.

In a plot without scaling of the distance, shown in Figure 7, all data for the cation-anion pair shown in Figure 5 are included. The positions of the second peaks in Figure 7 are not the same, while structures at the short distance overlap considerably. Similar slopes (marked by an oval) of $g(r)$ under different conditions mean that the PMF with $r^{-n}$ is common for all data. From eq 2 and the power law dependence of $W_{i j}$, we have

$$
-k_{\mathrm{B}} T \ln g_{i j}(\mathrm{r})=W_{i j} \propto r^{-n}
$$




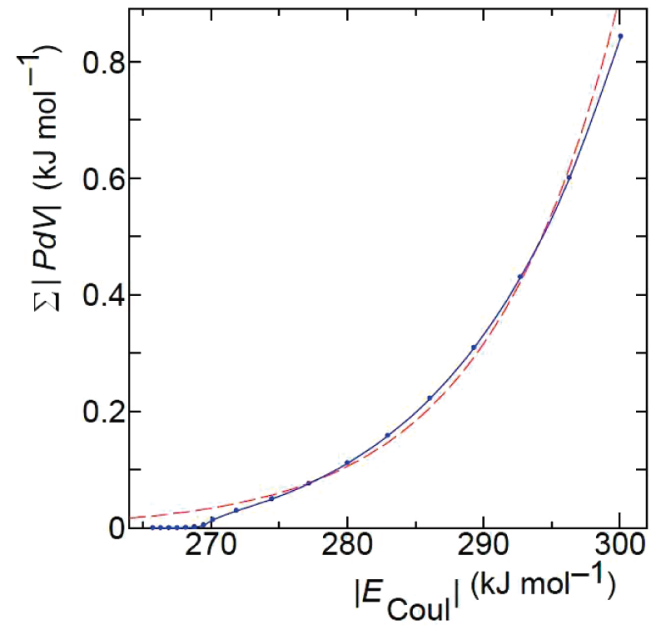

Figure 8. Relation between the Coulombic energy and the excess free energy of the system at $800 \mathrm{~K}$, both in absolute values. For the free energy, the value at $800 \mathrm{~K}$ under $\sim 0.1 \mathrm{MPa}$ is taken as the standard. The integral in eq 8 is replaced by the summation using a $\mathrm{d} V$ value of $5 \mathrm{~cm}^{3} / \mathrm{mol}$.

If one can substitute $r$ in eq 6 with $s(V / N)^{1 / 3}$ within a certain distance, the relation

$$
-\ln g_{i j}(r) \propto T^{-1} V^{-n / 3}
$$

is obtained, where $s$ is a constant. That is, $g(r)$ can be represented as a function of $T V^{\gamma}$, and a common $n$ governs the structures within the distance, where $r \approx s(V / N)^{1 / 3}$ holds. In this case, scaling can hold without self-similarity of the whole structure. Even if the scaling affects the relatively longranged structures or dynamics at long times, a scalingcontrolling region is not necessarily required to cover all regions. This is because the short-range softness determines the packing of the local structures, and this affects longrange structures and long-time dynamics. The common region with a similar slope is necessary to explain the existence of the common exponent under different conditions. Thus, these results seem to explain the existence of a common scaling exponent, although further examination of other systems might be necessary for a definite conclusion. The explanation may be also applicable to simpler systems because in a low density limit, the mean force potential becomes comparable to a bare potential.

The difference of the Helmholtz free energy $F$ of the system can be directly obtained from the integration of $P \mathrm{~d} V$ at constant temperatures as well as from ion-ion interactions

$$
\Delta F \equiv F_{1}-F_{0}=-\int_{v_{0}}^{V_{1}} P \mathrm{~d} V
$$

We calculated it for $T=800 \mathrm{~K}$, where the point with $T=$ $800 \mathrm{~K}$ and $0.1 \mathrm{MPa}$ was taken as a standard. We found good correlation between the excess free energy, $\Delta F$, of the system and the Coulombic energy term, as shown in Figure 8. In the figure, the integration in eq 8 was substituted for by the summations, that is $|\Delta F|=\left|\sum P \mathrm{~d} V\right|$. The relation between $\Delta F$ and the Coulombic energy looks like a power law except at the beginning $(<250 \mathrm{MPa})$. A power law fit is shown by a red dashed curve. The excess free energy can be related to the mobility of ions, although the connection with free-energy term and diffusion dynamics is not simple. According to dynamical density functional theory, ${ }^{50}$ the Helmholtz free-energy functional can be connected to the self part and distinct part of the van Hove functions. Further studies using these functions will be useful. Corresponding points on the master curve have comparable Coulombic energies, and similar structures and will have comparable free energy. This enables us to estimate the energy of the system at different temperatures using the master curve.

An argument for the Helmholtz-type free energy holds for the mean behavior of the system and is not necessarily applicable to the heterogeneous structure and dynamics in a disordered system, while the reason for the change in the slope of the master curves is heterogeneous dynamics, as already explained. For describing heterogeneous dynamics, extended statistics such as Tsallis statistics ${ }^{51}$ seems to be required. This is because the density profile formed by ions can have multifractal structures consisting of several exponents, as shown in the ionics in lithium silicate systems. ${ }^{36}$ Since the multifractal structure is connected with the Tsallis entropy rather than the Boltzmann entropy, further study including multifractal analysis will be helpful to understand the effect of the heterogeneity on the free energy and the dynamics. The multifractal walks in the ionic liquid have been found and discussed in previous works. ${ }^{52}$ Multifractality of the underlying structure of the density profile is important for consideration of the dynamics. These motions are concerned with the existence of different length scales, and therefore, the scaling is valid over wide length scales. This is consistent with the argument that the scaling behaviors include many-body terms and possible connections to the PMF.

Scaling is also applied to the motions of wide time scale regions. Strong correlation between relaxation times of the $\alpha$ process and the Johari-Goldstein $\beta$ process in the scaled behaviors in both the temperature and pressure dependence has been pointed out ${ }^{53,54}$ for glass-forming materials at temperatures above $T_{\mathrm{g}}$. Below $T_{\mathrm{g}}$, the relaxation times of both processes depends on the thermodynamic path in the formation of the glass. Here, the scaling has serious limits.

III.E. Comparison with Other Model Systems. The success of scaling in the real units is found to be based on the similar response of the system to temperature and pressure, which can be connected through the density-temperature variable in the form of $T V^{\gamma}$. The scaling law for the inverse power law (softcore) potential is known to hold, ${ }^{37}$ including dynamical properties in the phase space, ${ }^{38}$ and $n$ is connected to $\gamma$ of the system. Correspondence of the different states on the master curve in the present work is found for the diffusion coefficients not in the scaled units but in the real units. Obviously, the situation is different from that in ref 38, where the reduced units are used for dynamical properties such as the relaxation times. In the model soft-core system, the scaling is due to the self-similarity of the system, while the observed scaling law in the present work is valid for the temperature and pressure dependence within a system and related to the similarity of the structure of $g(r)$ for the small $r$ region in the real units. In the present work, we used the diffusion coefficients, $D$, for the thermodynamic scaling. In the experiments, scaling has also been confirmed for viscosity or the relaxation time for several systems. The corresponding quantity can be $D / T$ rather than $D$ based on the Stokes-Einstein law or the related fractional Stokes-Einstein law $^{55-57}$ of the form of $D \propto(T / \eta)^{s}$ or $D / T \propto(1 / \eta)^{t}$.

We have confirmed that similar scaling behavior is obtained when $D / T$ is used, albeit the deviation from the master curve is slightly larger.

Recently, Bailey et al. ${ }^{58}$ argued the correlations between pressure and energy in liquids based on the MD simulations of many model systems using NVT ensembles. They have reported 
that the correlation for the fluctuations in $U$ and $W$, where $U$ is a potential energy and $W$ is a virial of the system, is due to an effective inverse power law potential, and the correlation is destroyed by the Coulombic interactions. Our interpretation is also based on the effective potential, and therefore, some views are shared with them. The effect of the Coulombic force should cause the deviation from the dynamical scaling observed in the soft-core system as they discussed. However, scaling behaviors in the present work were not destroyed by the Coulombic term. This is because the scaling law in the present work holds for structures based on the ion-ion interactions rather than atom-atom interactions, and it is based on the similarity of the structure in real space. That is, the physical meaning of the scaling is not the same as the exact dynamical scaling law in the soft-core system. A many-body character of $g(r)$ and heterogeneity of the structures and dynamics also play important roles in our explanations.

Kanakubo et al. ${ }^{59}$ have reported the diffusion dynamics in the ionic liquid 1-butyl-3-methylimidazolium hexafluorophosphate ([BMIM]PF 6$)$ measured by NMR spin-echo technique. The system has relatively large cations. They found that when the values of the velocity correlation coefficients for a given isotherm were normalized relative to the corresponding atmospheric pressure values, they collapsed onto a single curve. On the other hand, Wojnarowska et al. $^{60}$ have reported some deviations from the scaling in the verapami hydrochloride, which is called a protic ionic liquid, by using a dielectric spectroscopy. The system contains quite larger cations compared with anions. In the work, they used a conductivity relaxation time instead of a structural relaxation time or diffusivity. However, if the mobility of anions and cations is considerably different, one cannot neglect the differences between conductivity and structural relaxations. The former will be more affected by the faster ions, while the latter will be more affected by the slower ions. Therefore, further examination of heterogeneous systems for dynamics and/or structures may be helpful to understand the observed breakdown of the scaling.

III.F. Applicability of Effective Potential Parameters for Coarse-Grained Dynamics. So far, several attempts to replace a long-range electrostatic potential energy by the short-range effective potential have been reported. Izvekov et al. ${ }^{61}$ have obtained a soft-core-type potential by the force-matching methods for water and $\mathrm{NaCl}$, where interaction potentials are determined on an atomic level. The success of this approach in different systems seems to be related to the generality of the scaling properties. Several levels of the coarse-grained potential parameters without a long-ranged Coulombic term can be obtained from the scaling properties and/or from the pair correlation function both by experiment and simulations, in principle. Applicability of effective potential parameters for coarse-grained dynamics is an interesting problem of further study. When it is used for modeling of multiscale systems, different levels of systems can be connected without changing the dynamic properties, if the coupling among different levels of systems is correctly taken into account.

The effective repulsive force found in the ion-ion interactions can have correlation with the repulsive term in eq 1. One may consider that the repulsive terms in eq 1 are directly related to the scaling exponent. From the result of the present work, we suggest that the repulsive parameters concerned with short distance for cation-anion pairs are the most important for the scaling. Therefore, it is worthwhile to check the scaling behavior by changing the values and combination of exponents in eq 1 in further works. The treatment using ion pairs seems to have merit for explaining both the importance of the Coulombic term and the role of the repulsive term in the scaling at the same time.

\section{Conclusion}

Scaling dynamics in ionic liquids exemplified by $\mathrm{EMIM}^{+}-\mathrm{NO}_{3}{ }^{-}$have been investigated by molecular dynamics simulations by using an all-atom model. For the diffusive dynamics of different temperature and pressure conditions, a master curve was obtained by scaling of $T V^{\gamma}$, with $\gamma=4.0$ for the cation and 3.8 for the anion. Dynamical slowing down represented by the empirical VFT equation or by the inflection of the temperature dependence of the diffusion coefficient is related to the change from longer- to shorter-length-scale dynamics. The master curve maintains these characteristics, and therefore, the scaling has many-body characteristics. Corresponding states having the same $T V^{\gamma}$ show a similarity in a Coulombic term as well as in structures represented by pair correlation functions. Actually, pressure-dependent behaviors of them were quite similar to temperature-dependent behaviors when we used the real units. We found that the exponent estimated from the potential of mean force is comparable to the $\gamma$. Considering both the simple liquids and the ionic liquids, the scaling of the dynamics is not necessarily directly related to the bare interatomic or intermolecular potential, while it appears to be related to the similarity of the structure and underlying effective potentials.

Acknowledgment. One of the authors (J.H.) thanks Prof. A. Ueda for helpful discussions. This research was partly supported by the Ministry of Education, Science, Sports and Culture, Japan, Grant-in-Aid for Scientific Research (C), 19540396, 2007-2009. The work performed at the Naval Research Laboratory was supported by the Office of Naval Research.

\section{References and Notes}

(1) Angell, C. A. Nature 1998, 393, 521.

(2) Ngai, K. L. J. Non-Cryst. Solids 2005, 351, 2635.

(3) Anderson, P. W. Science. 1995, 267, 1616.

(4) Chang, K. The Nature of Glass Remains Anything but Clear; The New York Times: New York, July 29, 2008.

(5) Sillescu, H. J. Non-Cryst. Solids 1999, 243, 81, and references therein.

(6) Lačević, N.; Starr, F. W.; Schrøder, T. B.; Glotzer, S. C. J. Chem. Phys. 2003, 119, 7372.

(7) Habasaki, J. J. Non-Cryst. Solids 2007, 353, 3956, and references therein.

(8) Tölle, A. Rep. Prog. Phys. 2001, 64, 1473.

(9) Roland, C. M.; Bair, S.; Casalini, R. J. Chem. Phys. 2006, 125, 124508.

(10) Casalini, R.; Roland, C. M. Phys. Rev. E 2004, 69, 062501.

(11) Pensado, A. S.; Pádua, A. A. H.; Comuñas, M. J. P.; Fernández, J. J. Phys. Chem. B 2008, 112, 5563.

(12) Michele, C. D.; Sciortino, F.; Coniglio, A. J. Phys.: Condens. Matter 2004, 16, L489.

(13) Coslovich, D.; Roland, C. M. J. Phys. Chem. B 2008, 112, 1329.

(14) Welton, T. Chem. Rev. 1999, 99, 2071.

(15) Earle, M. J.; Seddon, K. R. Pure Appl. Chem. 2000, 72, 1398.

(16) Wasserscheid, P.; Keim, W. Angew. Chem., Int. Ed. 2000, 39, 3772.

(17) Kitazume, T. J. Fluorine Chem. 2000, 105, 265.

(18) Del Popolo, M. G.; Voth, G. A. J. Phys. Chem. B 2004, 108, 1744.

(19) Morrow, T. I.; Maginn, E. J. J. Phys. Chem. B 2002, 106, 12807.

(20) Lopes, J. N. C.; Deschamps, J.; Pádua, A. A. H. J. Phys. Chem. B 2004, 108, 2038

(21) Hanke, C. G.; Price, S. L.; Lynden-Bell, R. M. Mol. Phys. 2001, 99, 801.

(22) Margulis, C. J. Mol. Phys. 2004, 102, 829.

(23) Habasaki, J.; Ngai, K. L. J. Chem. Phys. 2008, 129, 194501.

(24) Habasaki, J.; Ngai, K. L. Anal. Sci. 2008, 24, 1321.

(25) Wang, J.; Wolf, R. M.; Caldwell, J. W.; Kallamn, P. A.; Case, D. A. J. Comput. Chem. 2004, 25, 1157. 
(26) Case, D. A.; Darden, T. A.; Cheatham, T. E., III; Simmerling, C. L.; Wang, J.; Duke, R. E.; Luo, R.; Merz, K. M.; Pearlman, D. A.; Crowley, M.; Walker, R. C.; Zhang, W.; Wang, B.; Hayik, S.; Roitberg, A.; Seabra, G.; Wong, K. F.; Paesani, F.; Wu, X.; Brozell, S.; Tsui, V.; Gohlke, H.; Yang, L.; Tan, C.; Mongan, J.; Hornak, V.; Cui, G.; Beroza, P.; Mathews, D. H.; Schafmeister, C.; Ross W. S.; Kollman P. A. AMBER9; University of California: San Francisco, CA, 2006.

(27) Cang, H.; Li, J.; Fayer, M. D. J. Chem. Phys. 2003, 119, 13017.

(28) Berendsen, H. J.; Postma, J. P. M.; Van Gunsteren, W. F.; DiNola,

A.; Haak, J. R. J. Chem. Phys. 1984, 81, 3684.

(29) Morishita, T. J. Chem. Phys. 2000, 113, 2976.

(30) Habasaki, J.; Ngai, K. L.; Hiwatari, Y. Phys. Rev. E 2002, 66, 021205

(31) Ngai, K. L.; Moynihan, C. T. Bull. Mater. Res. Soc. 1998, 23, 51.

(32) Liu, X.; Jain, H. J. Phys. Chem. Solid. 1994, 55, 1433.

(33) Ngai, K. L. J. Chem. Phys. 1999, 110, 1576.

(34) Habasaki, J.; Ngai, K. L.; Hiwatari, Y. J. Chem. Phys. 2004, 120, 8195 .

(35) Vogel, H. Phys. Z. 1921, 22, 645. Fulcher, G. S. J. Am. Ceram. Soc. 1923, 8, 339 .

(36) Habasaki, J.; Ngai, K. L. J. Chem. Phys. 2005, 122, 214725.

(37) Hoover, W. G.; Gray, S. G.; Johnson, K. W. J. Chem. Phys. 1971, 55,1128 .

(38) (a) Hiwatari, Y.; Matsuda, H.; Ogawa, T.; Ogita, N.; Ueda, A. Prog. Theor. Phys. 1974, 52, 1105. (b) Ueda, A. Butsuri 2007, 62, 769, in Japanese. (c) Harris, J. Chem. Phys. 2009, 131, 054503.

(39) Nosé, S. Mol. Phys. 1984, 52, 255

(40) Nosé, S. J. Chem. Phys. 1984, 81, 511.

(41) Hattori, T.; Kinoshita, T.; Narushima, T.; Tsuji, K.; Katayama, Y. Phys. Rev. 2006, B73, 054203, and references therein.

(42) Kanakubo, M.; Umecky, T.; Hiejima, Y.; Aizawa, T.; Nanjo, H.; Kameda, Y. J. Phys. Chem. B 2005, 109, 13847.

(43) Kirkwood, J. G. J. Chem. Phys. 1935, 3, 300.

(44) Chandler, D. Introduction to Modern Statistical Mechanics; Oxford University Press: New York, 1987.
(45) Hansen, J. P.; MacDonald, I. R. Theory of Simple Liquids, 2nd ed.; Academic Press: London, 1990.

(46) Gray, C. G.; Gubbins, K. E. Theory of Molecular Fluids; Clarendon Press: Oxford, U.K., 1984, Vol. 1.

(47) Hirata, F.; Sato, H.; Ten-no, S.; Kato, S. In Computational Biochemistry and Biophysics; Becker, O. M., Mackerell, A. D., Jr., Roux,

B., Watanabe, M., Eds.; Marcel Dekker: New York, 2001; pp 419-439.

(48) Soper, A. Chem. Phys. 1996, 202, 295.

(49) Torrie, G. M.; Valleau, J. P. Chem. Phys. Lett. 1974, 28, 578.

(50) Archer, A. J.; Hopkins, P.; Schmidt, M. Phys. Rev. E 2007, 75, 040501.

(51) Tsallis, C. J. Stat. Phys. 1988, 52, 479.

(52) (a) Habasaki J.; Ngai, K. L. J. Phys. Soc. Jpn., Suppl. In press. (b) Habasaki, J.; Ngai, K. L. J. Chem. Phys. Submitted.

(53) Mierzwa, M.; Pawlus, M.; Paluch, M.; Kaminska, E.; Ngai, K. L. J. Chem. Phys. 2008, 128, 044512.

(54) Kessairi, K.; Capaccioli, S.; Prevosto, D.; Lucchesi, M.; Sharifi, S.; Rolla, P. A. J. Phys. Chem. B 2008, 112, 4470.

(55) Harris, K. R. J. Chem. Phys. 2009, 131, 054503.

(56) Habasaki, J.; Affouard, F.; Decamps, M.; Ngai, K. L. AIP Conf. Proc. 2008, 982, 154.

(57) Funazukuri, T.; Kong, C. Y.; Kagei, S. J. Supercrit. Fluids 2008, 46, 280, and references therein.

(58) (a) Bailey, N. P.; Pedersen, U. R.; Gnan, N.; Schrøder, T. B.; Dyre, J. C. J. Chem. Phys. 2008, 129, 184507. (b) Schrøder, T. B.; PedersenU. R.; Dyre, J. C. E-print arXiv:0803.2199.

(59) Kanakubo, M.; Harris, K. R.; Tsuchihashi, N.; Ibuki, K.; Ueno, M. J. Phys. Chem. B 2007, 111, 2062.

(60) Wojnarowska, Z.; Paluch, M.; Grzybowski, A.; Adrjanowicz, K.; Grzybowska, K.; Kaminski, K.; Wlodarczyk, P.; Pionteck, J. J. Chem. Phys. 2009, 131, 104505.

(61) Izvekov, S.; Swanson, J. M. J.; Voth, G. A. J. Phys. Chem. B 2008, $112,4711$.

JP911157K 